The architectural publications in latin america: profiles to understand their path

\section{| RESUMEN |}

Estos párrafos presentan la variedad de perfiles y de fisonomías de las revistas de arquitectura latinoamericanas presentes en el siglo $x \times$. Considera la metamorfosis informática a la que nos somete la realidad de esta nueva centuria propone un análisis de las revistas relevantes en el continente y, si bien no aspira a desarrollar una relación exhaustiva, presenta la coyuntura a la que nos enfrentamos los investigadores, dada la importancia unívoca de este material documental.

\section{| ABSTRACT |}

These paragraphs present the variety of profiles and faces of Latin America's magazines of architecture in the twentieth century. The text considers the information technology metamorphosis to which we subject the reality of this new century, and offers an analysis of relevant journals on the continent and, while not aimed at developing an exhaustive relation, presents the economic situation we researchers are facing given the unequivocally importance of this documentary material.

\title{
Las publicaciones de arquitectura en Latinoamérica: perfiles para comprender su trayectoria
}

\begin{abstract}
quellos que recorremos estas líneas Aconocemos ampliamente la capacidad para informar y referenciar la arquitectura del modo en que -casi únicamente- lo hacen las páginas de las revistas especializadas. Acordamos que consolidan el testimonio de una acción relativa al ejercicio y la valoración profesional y, seguramente, podemos medir hoy sus magnitudes con mayores perspectivas que aquellas que tuvieron sus autores al momento de editarlas.
\end{abstract}

Pero, ¿̇de qué hablamos cuando hablamos de una revista de arquitectura? Parece tratarse de una pregunta de simple respuesta, pero es de explicación compleja si consideramos todos los factores que concurren a su conceptualización. Podríamos definirla, en tanto medio de comunicación, como aquella «publicación periódica que se dedica a la arquitectura o a sus dominios anexos: el edificio, la edificación, la construcción, aspectos inmobiliarios, trabajos públicos y de la misma manera incorpora temas de urbanismo, monumentos o edificios arqueológicos y hasta de decoración arquitectónica; también recurre a la historia de la arquitectura y aspectos del patrimonio» ${ }^{[1]}$.

Sin lugar a dudas, es fuente reveladora esencial para la profesión y su creciente demanda de consulta en las bibliotecas testimonia su importancia documental; empero, una constante se nos impone: las herramientas bibliográficas sobre la prensa arquitectónica son escasas y los métodos para el estudio de este periodismo especializado, son algo imprecisos e inciertos y muestran una incipiente formalización dentro de sus líneas de investigación.

Los arquitectos no tenemos la culpa de ello, en el decir de Mirta Varela, «la relación de los medios de comunicación con la Historia está repleta de confusiones y ambigüedades», por ello «la importancia de los medios de comunicación en la sociedad contemporánea es indiscutible y las hipótesis interpretativas del rol que cumplen, apelan a la historia sin excepción: las transformaciones técnicas, los cambios en la socialidad, o de las relaciones entre lo público y lo privado, suelen comprenderse desde

* Arquitecta (UBA). Investigadora Adjunta, CONICET y Coordinadora Técnica del Centro de Documentación de Arquitectura Latinoamericana (CEDODAL) a cargo de las exposiciones y ediciones producidas por esa institución. Directora del Centro de Investigaciones del Imaginario Visual en la Facultad de Arquitectura de la Universidad de Belgrano donde también ejerce como titular de Historia de la Arquitectura. Miembro del Comité del ARLA (Asociación de Revistas de Arquitectura Latinoamericana) con sede en Chile. Autora de numerosos artículos y de libros vinculados a la historia de la fotografía y de la arquitectura a través de las revistas especializadas. Ha sido distinguida con el premio al Proyecto de Investigación en la VI BIAU (Lisboa, 2008) y en la Universidad de Belgrano (2009) además de becaria del Getty Center y del Fondo Nacional de las Artes en más de una ocasión.

[1] Leniaud, Jean Michel; Bouvier, Béatrice. Les périodiques d'architecture. XVIIle- XXle siècle. Recherche d'une méthode critique d'analyse. Paris: Études et rencontres de l'École des Chartres, 2001. 
1. Portadas de revistas de arquitectura latinoamericanas.

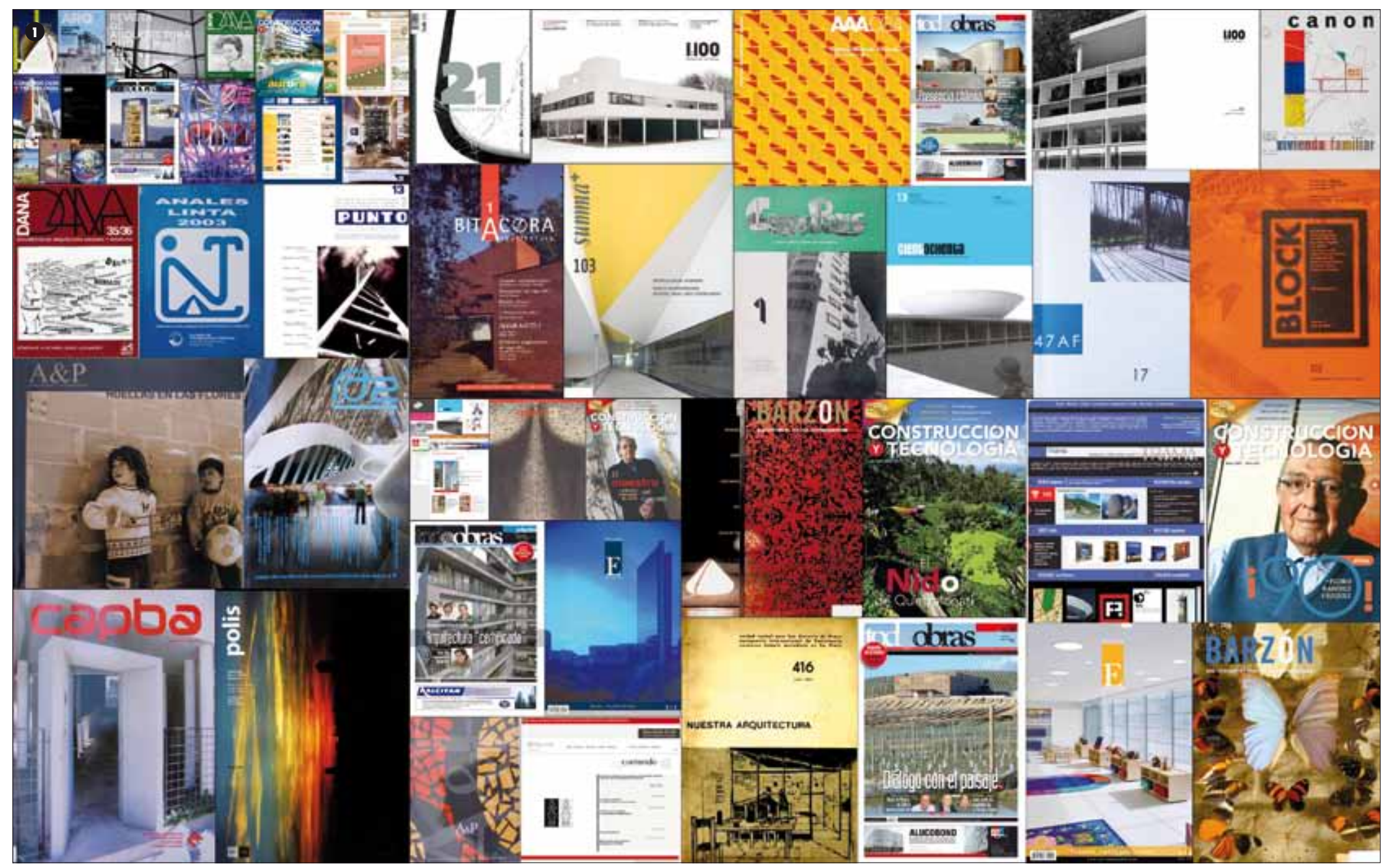

el análisis del momento de su emergencia del contraste con las formas previas y su evolución» ${ }^{[2]}$

Conjuntamente con esta ansiedad disciplinar conviven numerosas dificultades que se presentan a la hora de consultar colecciones completas y ello se debe a hechos que radican en la propia naturaleza de las revistas. Entre los factores que interrumpen una sistemática investigación, priman: la vida efímera de muchas de ellas, los cambios de nombre y de formato dentro de una misma editorial, la repetición de títulos en distintos países, la discontinuidad de las ediciones, los problemas de circulación derivados de que muchas revistas fueron editadas fuera de los circuitos comerciales y por distintos tipos de entidades profesionales -fuesen estas académicas o institucionales-, además de los avatares económicos a los que la industria gráfica de nuestros países fue sucesivamente sometida.

El ámbito globalizante actual permite nuevas formas de transmisión de la información y esta coyuntura nos permite verificar que, más allá de los modelos recientes, se mantienen ciertos patrones vigentes en la difusión de

[2] Varela, Mirta. «Medios de comunicación e industrias culturales: historias nacionales y problemas globales». Documento presentado en el XXX Congresso Brasileiro de Ciencias da Comunicação. São Paulo: Intercom Sociedad Brasileira de Estudios Interdisciplinares de Comunicação, 2007.

contenidos que estuvieron también antes, durante y perduran hasta hoy. Es que las publicaciones respondieron tradicionalmente a los criterios de sus editores y mientras las comerciales se nos presentan como las más flexibles, adaptándose a una mirada versátil y atentas a los requerimientos del mercado, en otras verificamos que se constituyeron en voceras de tendencias arquitectónicas o de marketing; algunas otras expresaron segmentos particulares como las originadas en centros estudiantiles o en organismos gremiales y también muchas veces, sobre todo entre las más antiguas, la información sobre la arquitectura la podemos rastrear a través de publicaciones artísticas o literarias que incluyeron páginas específicas dedicadas a nuestra disciplina. 


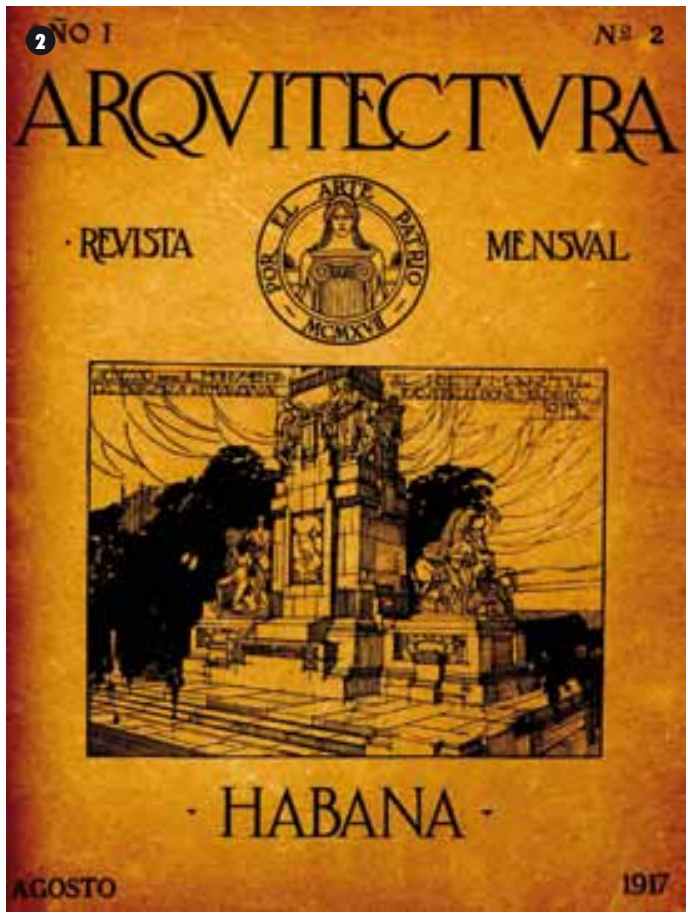

De estos análisis tratarán estos párrafos, apuntando apenas a un repaso -con omisiones involuntarias- de la variedad de perfiles y de fisonomías editoriales de la arquitectura latinoamericana del siglo xx a las que nos enfrentamos, haciendo especial hincapié en la metamorfosis informática a la que nos somete la realidad de esta nueva etapa, proponiendo un análisis de esas ediciones aunque sin encarar una relación exhaustiva que escapa al espacio destinado en esta publicación. De esta manera y de cara al siglo xxı, tal vez, se nos facilite una comprensión más amplia del universo cultural y arquitectónico que nos dejó la centuria anterior, expresada no solamente en la selección de lo que se publicaba, sino también en la opinión y orientación de quienes editaron esos volúmenes, facilitándonos la visión de otros aspectos de la arquitectura de ese tiempo.

\section{LOS PERFILES EDITORIALES \\ DEL CONTINENTE}

Entre las revistas más antiguas y con perdurabilidad continental tenemos la REVISTA DE ARQUITECTURA de la SAU (Sociedad de Arquitectos de Uruguay), de frecuencia mensual y editada entre 1914 y 1940, las argentinas ARQUITECTURA
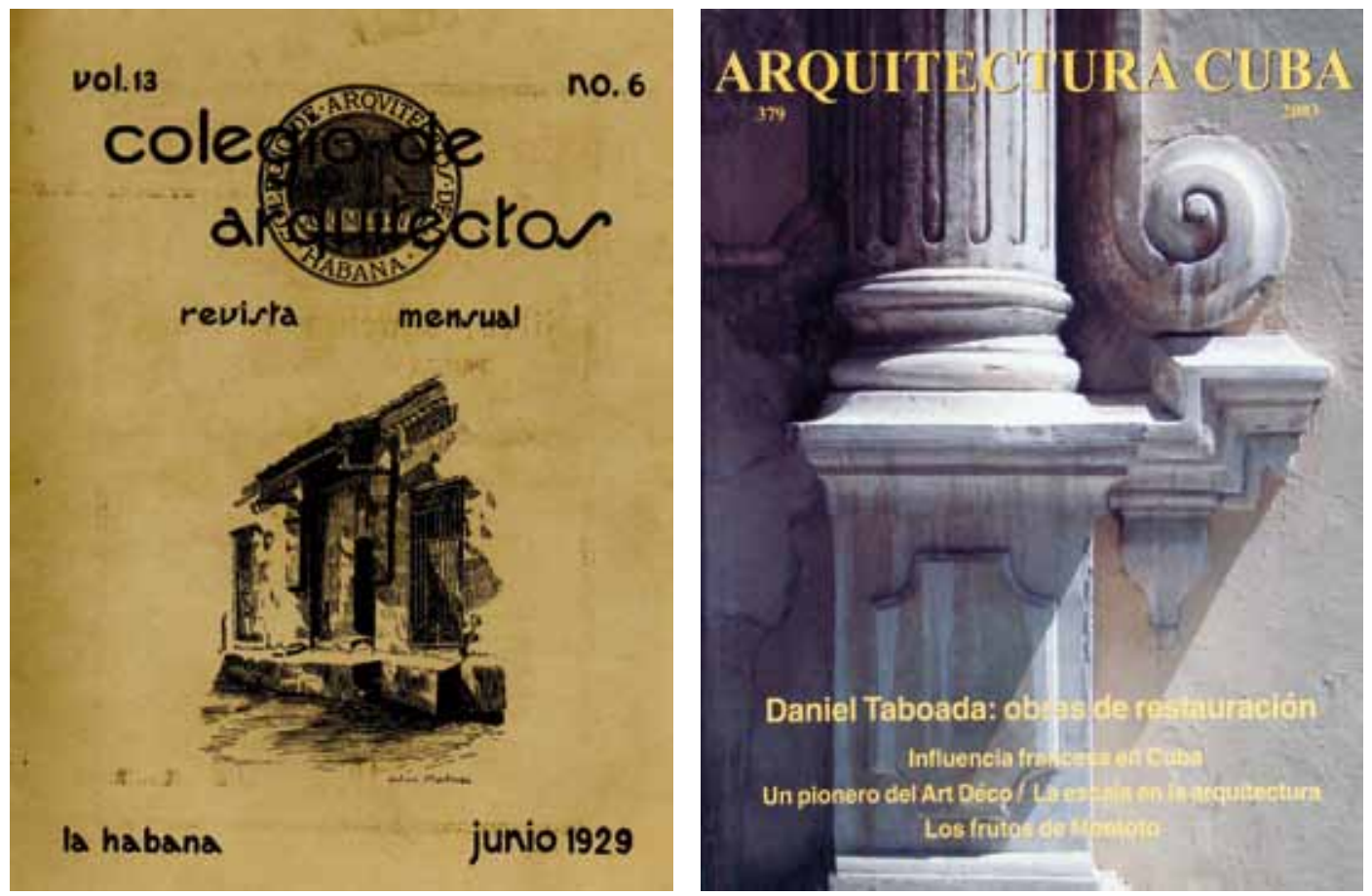

(1904-1916) como desprendimiento de la REVISTA TÉCNICA (1895-1910), y desde la ciudad de Rosario con una visión sudamericana que más tarde cumplirán otras revistas de la profesión encontramos EL CONSTRUCTOR SUDAMERICANO (1911) cuyos antecedentes se remontan a otras publicaciones que le dieron origen: LAS Artes EdILICIAS (1908) y luego El ArQUITECTO CONSTRUCTOR (1919).

En este renglón de las primeras también figura ARQUITECTURA CUBA, vigente desde 1917, a la cual además le caben las características de una edición que cambió en ocho ocasiones de nombre, manteniendo idéntica pertenencia institucional y presentándonos numerosas dificultades a la hora de organizar una colección. Podemos atender aquí también los casos de revistas de arte devenidas en publicación de arquitectura, como el ejemplo de Áurea (Argentina) -que en realidad nació como ARQUITECTURA y ARTE DECORATIVO- y, a partir de la séptima edición, presentó su nuevo nombre al cual sumó en tipografía menor, debajo del título ÁUREA, la frase "Arquitectura y arte decorativo» y que más tarde, además, remplazaría por Revista Mensual de Todas las Artes. Asimismo, en Chile la vinculación entre las dos disciplinas se detecta en las publicaciones ArTE y ARQUITECTURA publicada entre 1922 y 1923 y-repitiendo el título argentino-, en ARQUITECTURA y ARTE Decorativo (1928-1929).

Frente a este panorama, la continuidad y permanencia en el medio editorial son aspectos para destacar en la trayectoria de algunas de las publicaciones del continente. En este sentido, encontramos ediciones comerciales afines a un estudio profesional o promovidas por empresas, con ejemplos como ArQUITECTURA MÉxICO, creada y dirigida por Mario Pani cuando promediaba la década del '30, logrando 119 ediciones; también El Arquitecto Peruano, fundada por Fernando Belaúnde Terry, quien publicó 354 números y fue valorizada por fomentar la arquitectura histórica de ese país alentando el rescate de sus aspectos vernáculos. $\bigcirc$ bien el ejemplo de la colombiana PrOA que mostró en sus 420 números, de la mano de Carlos Martínez, no solo calidad en su información sino que incluyó relevantes planteamientos teóricos y aspectos críticos de la arquitectura de su país. Particular atención merecen las ediciones brasileras, ejemplos como Módulo -editada entre 1955 y 1989, desde Rio de Janeiroque reflejaba en sus páginas la promoción personal del propio Oscar Niemeyer y BRASILIA (1957-1988), a cargo de la NOVACAP, que 
3. La Revista TÉCnICA editada entre 1895 y 1910 de Buenos Aires.

en su primera etapa documentó eficazmente el proceso constructivo de la ciudad que le daba su nombre. Pero no fueron las únicas, desde Belo Horizonte, se destacó Brasil ARQUITETURA Contemporânea (1953-1957) de Edições Contemporâneas. En este listado existen dos ejemplos argentinos emblemáticos: NUESTRA ARQUITECTURA, de regularidad exitosa entre 1929 y 1986, con 523 ediciones, ofreciendo enfoques de la modernidad local y el caso de Summa, que llegó con su principal producto a las 300 publicaciones, además de instalar otros títulos simultáneos a la revista principal, que facilitaron nuevos caminos a la comprensión de la arquitectura nacional a través de la teoría de la arquitectura (SUMmarios, SUMma Universitaria y Summa Colección Temática) y también, entre las de largo aliento y que aún perduran en el mercado, no puede dejar de señalarse la actividad de la colombiana ESCALA, a cargo de una empresa familiar que hoy también nos acerca otros productos vinculados a la profesión desde Bogotá.

Existen también ejemplos con manifiesta transitoriedad en la permanencia editorial, un renglón que generalmente está ocupado por las revistas universitarias pues, tanto como las institucionales, se ligan a la voluntad política de las autoridades de turno y el interés que en su promoción editorial se hayan propuesto. Podemos revisar algunos casos: PUNTO, desde Caracas, perteneciente a la Universidad Central de Venezuela, GáveA de la Universidad Católica de Rio de Janeiro y Fau de la Facultad de Arquitectura y Urbanismo de Montevideo. Entre estas distintas fases observamos otras producciones débiles por su durabilidad y paralelos a los citados; por ejemplo en Montevideo, y dentro de la misma Facultad, se editaban simultáneamente ANALES, luego la REVISTA DE ARQUITECTURA y también la del Instituto de Urbanismo.

También en la por entonces Facultad de Arquitectura, de la UBA, existió CANON. Órgano Oficial de la Fau, con una propuesta trimestral que lanzaba su primer número el 30 de diciembre de 1950, pero para el segundo hubo que aguardar a febrero de 1953. Sus directores reconocían en su presentación que el proyecto editorial llevaba dos años de "laboratorio» y su propuesta presentaría la cultura arquitectónica y urbanística que reflejaba la «acción social» acorde al momento políitico del país. Sin embargo, cuando sus páginas se refieren a la «Arquitectura Moderna», recurre a un material ya editado de otras revistas contemporáneas locales o extranjeras como Modern Bauformen, Revista de Arquitectura, TECNÉ, etc. La actual FADU se constituye en paradigma de quehaceres editoriales de serias intenciones y breve vida pues si bien coexisten distintas ediciones como ÁrEA - a cargo de la Secretaría de Investigaciones- y ANALES - del Instituto de Arte Americano (IAA)-, y aunque el claustro académico actual reúne seis carreras universitarias vinculadas al diseño, no ha podido consolidar la edición de una revista abarcante de la expresión del ámbito educativo que reúne a poco más de 27.000 alumnos. Nada más, valga como ejemplo la nunca presentada NiveL Más, con una propuesta concursada a fines de 2006 y que, por falta de presupuesto, vio coartadas todas sus iniciativas de publicación. Se destacan por número de títulos editados desde centros universitarios chilenos, por mencionar algunas, las publicadas desde la Universidad de Chile como TÉCNICA Y CREACIÓN (1960-1967), Ágora (1986-1987) o la Revista de ArQuitectura; en tanto, desde la Pontificia Universidad Católica, se publicó ARQ. ARquitectura, Diseño y URBANISMO (desde 1980), los CuADERnOS desde la Universidad de Valparaíso o los del mismo nombre en la Universidad Católica del Norte; también ARQUITECTURAS DEL SUR desde Concepción con la Universidad del Bío-Bío o la REvISTA_180, una publicación de la Facultad de Arquitectura, Arte y Diseño de la Universidad Diego Portales que se edita desde 2006.

Desde el interior de la Argentina, sobresalen las publicaciones de la provincia de Santa Fe, como Pous, vigente desde 1998 en la Universidad Nacional del Litoral y con un contenido que integra investigaciones académicas e incluye resúmenes de tesinas de graduación, producciones de estudiantes en el marco de programas de iniciación a la investigación y reseña la actividad institucional en una clara expresión de pluralismo académico. En este orden también se ubica la rosarina A\&P ARQUITECTURA Y PLANEAMIENTO, editada desde 1963 y con llamativos cambios en su formato a partir de su edición 17 cuando sus páginas eran dedicadas al $80^{\circ}$ aniversario de creación de la FAU. De carácter universitario también es 47 AL Fondo, de la ciudad de La Plata, que en su título

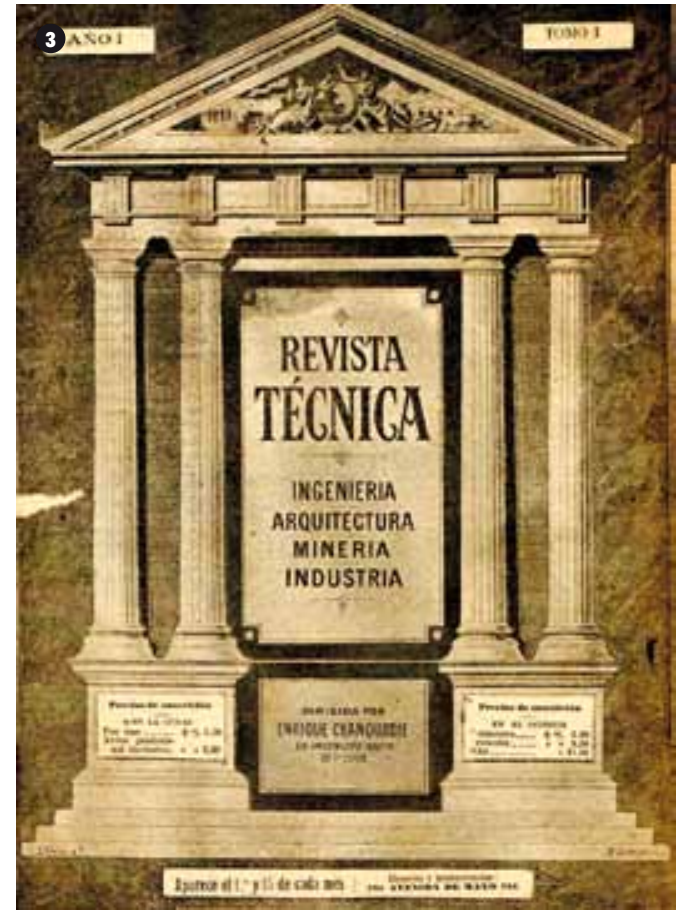

juega con la ubicación del centro educativo[3], se publica desde 1997 con una valorada calidad en la puesta en página y en su contenido.

En este mismo ámbito también existen las revistas promocionadas por los centros de estudiantes, quienes muchas veces han sido el germen de publicaciones destacadas. De hecho así nació la ReviSTA DE ARQUITECTURA de la Sociedad Central de Arquitectos y, en el mismo apartado podemos citar a CEDA -de Uruguay-, TAlLer -de los años cincuenta desde Caracas y que surgió como promotora de difusión del Movimiento Moderno- actualmente continuada por ENTRERAYAs, y las efímeras RePLANTEO o FONDO Y FIGURA, ambas de la UBA.

A partir de la década del '30 surgieron las publicaciones relacionadas con investigaciones académicas que, además de circular en grupos reducidos y sin espíritu comercial, fueron producto de institutos o departamentos específicos dentro de las mismas universidades. En este listado caben ANALES del IAA, grupo

[3] Las calles de la ciudad de La Plata, no se consignan con nombres propios, sino con números pares en un sentido e impares en sus perpendiculares. 


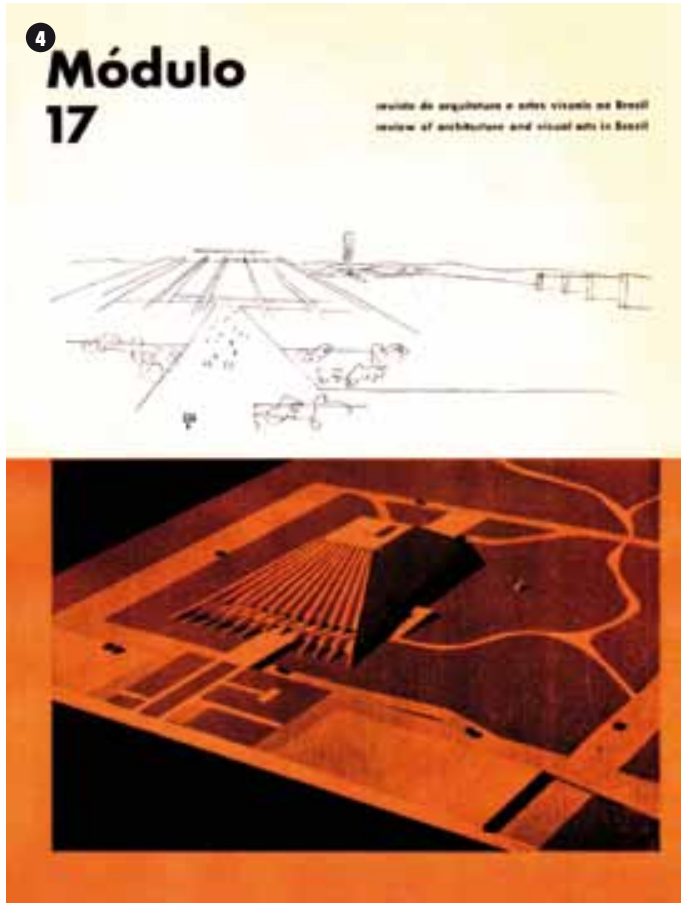

que editara también en diversos formatos los números monográficos de CRíticA -acordes a las investigaciones presentadas en su ámbito-, y también de su cuño los CuAdernos DE HISTORIA, luego $\mathrm{CH}$. Originadas en otros contextos podemos citar APUNTES, de la Universidad Javeriana de Bogotá, si bien discontinuada fue retomada hace poco tiempo con la idea de alentar la preservación del patrimonio y la historiografía latinoamericana; BARROCO, de Minas Gerais, de publicación interrumpida y la revista MarINa WaISMAN, en el ámbito de la Facultad de Arquitectura de la Universidad Católica, organizada por un grupo de profesionales en la ciudad de Córdoba a partir del fallecimiento de la autora a quien honra su nombre. En las universidades privadas argentinas como la de Belgrano, se publican los Documentos de Trabajo que edita números monográficos de frecuencia estable y reuniendo en su interior una serie de investigaciones comunes a un tema, pero no siempre exclusivo de arquitectura; por su parte, la Universidad Torcuato di Tella, a través de su Centro de Estudios de Arquitectura Contemporánea, edita desde 1997, Block, Revista de Cultura de la Arquitectura, la Ciudad y el Arte dirigida por el arquitecto Liernur con un comité editorial formado por diferentes entidades académicas

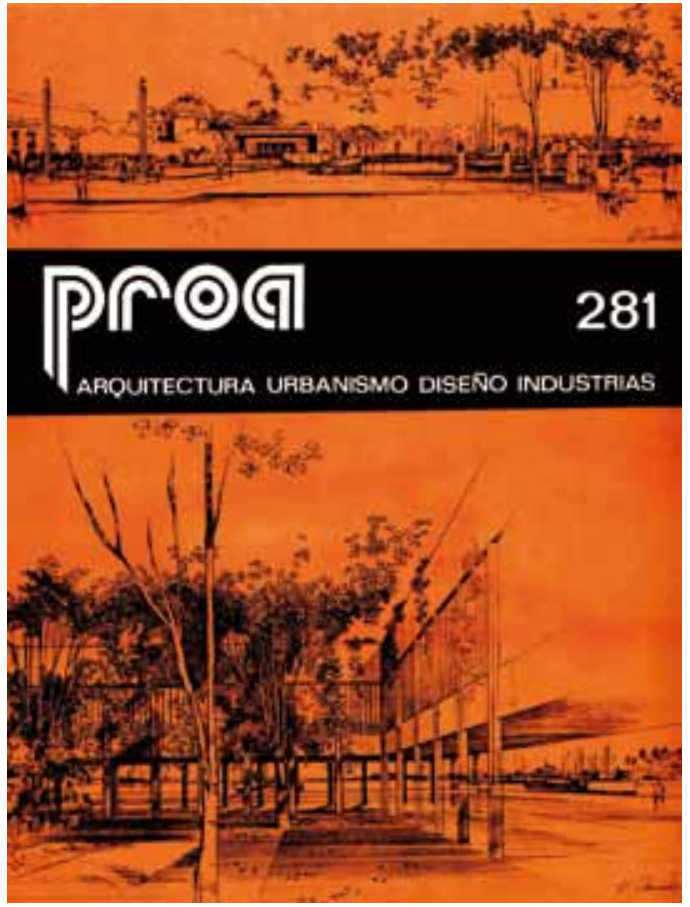

y, a pesar de la anhelada frecuencia semestral, desde 2004 a la fecha ha publicado ocho ediciones enfocadas «en las operaciones sobre la arquitectura, la ciudad y el territorio». En este mismo espacio de la teoría y de la investigación, no puede soslayarse la revista DANA, que nació en 1973 en el seno del IAIHAU[4], y la cual pese a los esfuerzos por mantener su continuidad es dificultosa su permanencia en el mercado por falta de sostén económico. Acciones similares suceden en México, a partir de la década del '80, cuando desde la UNAM se publicaban las series Cuadernos de Arquitectura Mesoamericana, Cuadernos de Arquitectura VirReinal, Cuadernos DE URBANISMO y CUADERNOS DE INVESTIGACIÓN Y

DOCENCIA. Sus ediciones fueron suspendidas para formar una única revista que decidió integrar sus contenidos bajo el nombre de la actual BITÁCORA ARQUITECTURA desde su Facultad de Arquitectura. En este apartado también sobresalen las chilenas Ars. Revista LatinOAmerICANA DE ARQUITECTURA (editada entre 1978 y 1991) a cargo del grupo CEDLA cuyo propósito era traer al conocimiento público las gestas continentales de nuestra modernidad arquitectónica; también EURE. Revista LatinOamericana de Estudios Urbano
Regionales cuya edición desde el Instituto de Estudios Urbanos de la PUC a partir de 1971 no ha cesado, si bien sus contenidos ya pueden leerse directamente desde la web.

Observando detenidamente muchos de los contenidos de estas páginas, sobresale la articulación que nuestra disciplina posee con las distintas especificidades del área de diseño que en ocasiones favoreció la disciplina y, en otras, apuntó a revalorizar la edición como objeto más que a su contenido. Encontramos en este conjunto a Lina Bo Bardi desde São Paulo, cuando en la revista HABITAT incluyó hasta el diseño de muebles 0 , en la década del '40, la mexicana ARQUITECTURA y LO Demás con su docena de ediciones impactaba con su diseño gráfico. Más acá en el tiempo, la chilena DisEÑO editada entre 1990 y 1998; O, desde 2004, la edición uruguaya DESPACIO cuya revolucionaria propuesta nos presenta visiones particulares, fragmentadas, y especialmente diagramadas para cada artículo, complicando tanto su asimilación que no podemos menos que aseverar su nombre: se debe leer muy «des-pacio».

Los casos argentinos que incorporaron otros actores del diseño al espíritu de la arquitectura surgieron a mediados de siglo xx, con Tomás Maldonado a la cabeza de la revista Nueva VISIÓ́n. Revista de cultura visual, una plaza que luego del cierre de esta quedó vacante y recientemente fue retomada por BARZON - a cargo de la empresa que realiza SummA +- con páginas de excelente calidad y mostrando el diseño del espacio a través de otras disciplinas que engloban las nuevas tendencias como los paisajes y el diseño de muebles o de interiores, entre otras.

Ya se han referenciado las sociedades de arquitectos de algunos países y dentro de este ámbito, debe destacarse la permanencia de las revistas que edita el Colegio de Arquitectos de Chile y la Sociedad Central de Arquitectos porteña, ambas con notorias mejoras en su calidad editorial con el correr del tiempo. También han hecho lo propio algunos Colegios de Arquitectos argentinos, mostrando voluntad al fomentar nuevos espacios editoriales: tales son

[4] Instituto Argentino de Investigaciones de Historia de la Arquitectura y del Urbanismo. 
los casos de RD2 del Colegio de Arquitectos de la provincia de Buenos Aires -distrito 2 Banfield-, acercando nada menos que 3.500 ejemplares de distribución gratuita para sus miembros como a las instituciones que así lo requieran, con páginas que, sin aspirar a ser temáticas, finalmente lo terminan siendo. De la mano del mismo director editorial que RD2 tenemos a CAPBA del Colegio de Arquitectos de la provincia de Buenos Aires en un espacio exclusivo para los concursos que la organización fomenta como parte del Programa Comunicacional, implementado desde el boletín digital en el año 2008.

Si nos referimos a las revistas comerciales y sus diferentes ópticas contamos, en el contexto argentino, con HáBITAT que está en la calle desde 1993 y se dedica a la preservación, el reciclaje, la conservación y restauración del patrimonio cultural; también Summat, que al año de la desaparición de su par casi homónimo -aquella publicación insigne en nuestro ámbito durante 40 años-, su edición ocupó el mercado vacante en un momento difícil pero acertado, con ediciones trimestrales de excelencia dedicadas a la reflexión arquitectónica desde los más distintos e interesantes puntos de vista. En el mismo renglón, y de carácter más técnico, tenemos ENTREPLANOS dirigida por el arquitecto Gustavo Di Costa; la más pequeña por su formato, nacida en 2004 de la mano de estudiantes de arquitectura y de diseño gráfico, denominada 1:100 con la particularidad de mostrar en cada edición una única obra de arquitectura reconocida, poniendo el acento en la documentación gráfica en la escala que da el nombre a la publicación e incorporando opiniones, además de fichas técnicas de la misma obra y la más nueva, PLOT, surgida como nueva plataforma para la difusión de las prácticas y pensamientos que hacen al campo cultural de la arquitectura contemporánea.

\section{DE CARA AL SIGLO XXI}

Frente a la realidad que compromete hoy la profesión en términos de actualización, una faceta que tomó entidad en lo que a publicaciones especializadas se refiere, son las secciones de arquitectura incluidas dentro de los periódicos. Por ser los más cercanos, y consciente de que no son los únicos en el continente, en los párrafos siguientes son comentados los ejemplos argentinos. El caso de la Sección Arquitectura del diario CLARín, nacida a inicios de los años '80 e incluida primero como páginas sueltas, luego se transformó en sección semanal y, a partir de 2002 y con la misma frecuencia, se independizó de la tirada periódica como producto opcional adoptando el nombre de $A R Q$, además de sumar a su contenido otros aspectos del diseño como el industrial y el gráfico y contemplar temas relacionados con la construcción, aspectos inmobiliarios y costos de materiales. La línea de publicaciones de su editor, el grupo Clarín, se complementa con la realización de guías de ciudades, libros dedicados a arquitectos, etc. El otro ejemplo es la sección que el diario LA NAción reservó para la arquitectura publicando los días miércoles durante 40 años (inicialmente como tapa o, a lo máximo, dos páginas de la sección de clasificados); su iniciador fue el periodista Daniel Viacava y contempla en su historia varios cambios de tamaño: fue "sábana» hasta 1994 cuando se transformó a tabloide y a color, en 2000 volvió al tamaño de origen e incrementó la cantidad de páginas con un mínimo de ocho, en 2006 volvió a tabloide y, a partir de abril de 2009, el impreso solo edita los primeros lunes de cada mes, mientras la versión online se actualiza semanalmente cada miércoles. En formato de periódico, pero concebida diferente por su distribución gratuita, tenemos TODOOBRAS, dirigida y editada por el colega argentino Tomás Dagnino, este nuevo formato editorial nació en Chile en 2002 para mostrar mensualmente la arquitectura de ese país, dándole cabida a la versión argentina a partir de 2004 y en los últimos años también a su relato desde Colombia.

Y así como este último modelo no encuadra directamente ni en la versión de periódico, ni en el de revista, gracias a los avances informáticos de hoy disfrutamos de otras posibilidades de lectura a través de distintos y novedosos formatos. Dentro de estas líneas se destaca la digitalización de revistas de arquitectura mexicanas emprendidas por la UNAM bajo el nombre COLECCIÓN RAíCES DIGITAL, coordinada por Carlos Ríos Garza, a través de la cual se han recuperado en formato de disco compacto los artículos publicados en las páginas de Anuario de la Sociedad de Arquitectos Mexicanos, Arquitectura y lo demás, El ArQuitecto y TrazA, Temas de ArquiteCtURA y URBANISMO, en una

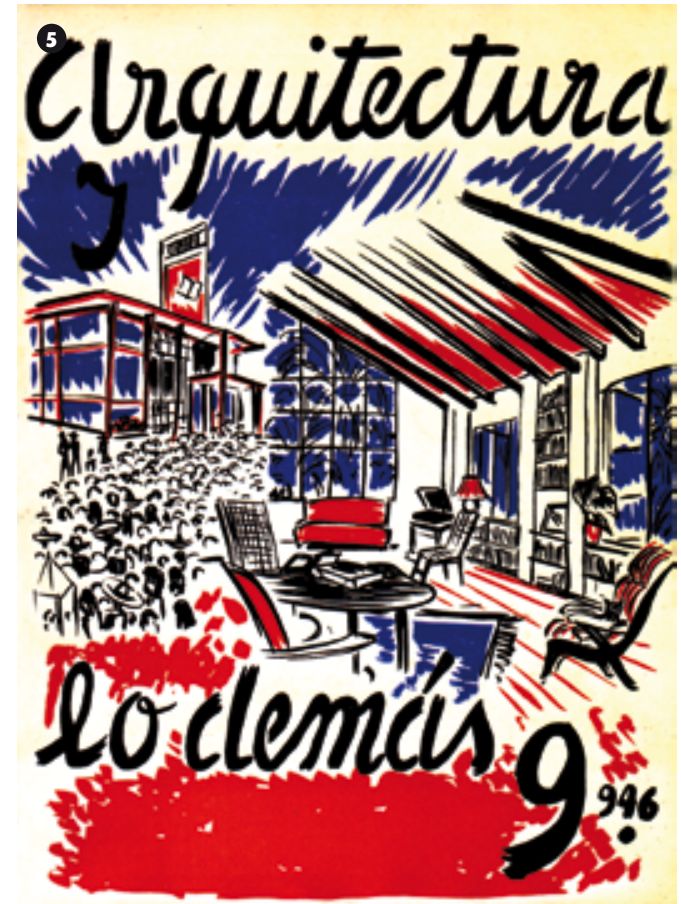

acción imitable por demás que rescata el corpus documental que encierran estas series hemerográficas.

Dentro de las novedades a las que tendremos que habituarnos, no pueden eludirse aquí los comentarios acerca de las versiones on line que hoy por hoy actualizan y facilitan la noticia al instante del quehacer profesional, sobre todo con aquellas revistas que además de publicarse a través de los canales habituales, lo hacen en Internet. Entre ellas, las peruanas Ac. Arquitectura Contemporánea o Arkinka que nos permite leer sus ediciones desde 1995; en Colombia, AxxIs desde 2007 y la propia EsCALA; la venezolana Entre RAYAS; desde México, Diseño y SOCIEDAD de la UAM-Xochimilco que atiende los campos de los diseños y sus problemáticas; o la paraguaya AIA, creada en 1993 como revista de arquitectura ingeniería y artes online, y $\mathrm{CENTROH}$ perteneciente a la Organización Latinoamericana y del Caribe de Centros Históricos (OLACCHI), bajo la conducción de Fernando Carrión y que busca ser un espacio de debate, actualización, investigación y consulta para académicos, formuladores de políticas y de opinión pública sobre las ciudades entendidas como entidades históricas, de frecuencia cuatrimestral en sendas presentaciones: papel e Internet. 
Asimismo, destacan en versiones únicamente digitales las brasileras ARQUIRAZ y de la mano de la tarea de Abilio Guerra, el portal Vitruvius. A ellas se suman las argentinas, 30-60. CuAderno LATINOAMERICANO DE ARQUITECTURA (aludiendo a los paralelos que limitan el continente) y que refleja las críticas, teoría y actualidad con cuadernos temáticos en cinco secciones, agendas, cursos, etc., además del Boletín Cedodal actualizado trimestralmente reportando las actividades de la entidad.

Más allá del somero detalle presentado, es cierto que el interés por las publicaciones especializadas de nuestro quehacer ha crecido mucho en los últimos años. Falta mucho por hacer, pero en este camino, sería injusto no mencionar los diversos proyectos de investigación realizados tanto en ámbitos universitarios sudamericanos como europeos y que entienden a las revistas de arquitectura como fuente documental imprescindible, revelando una actitud que trasciende el mero valor cuantitativo y cualitativo que ofrecen estas ediciones.

\section{CUESTIONES PENDIENTES Y ESFUERZOS CONCRETOS}

Cualquiera de los casos revisados hasta aquí nos señala cabalmente rumbos posibles a seguir, pero asimismo alimentan la expectativa académica y profesional para que en Latinoamérica cada biblioteca de arquitectura cuente al menos con las colecciones completas de las revistas editadas en su propio país. Dadas las circunstancias en que se encuentran la mayoría de los centros documentales del continente, este hecho favorecería enormemente la comunicación regional y facilitaría el acceso a un fondo hemerográfico completo y homogéneo hasta ahora inexistente. A la luz de los análisis resulta imprescindible apuntalar el diseño de políticas biblioteconómicas que revaloricen estas publicaciones $y$, a partir de ellas, se allanen los caminos para esa lectura transversal tan necesaria a nuestra arquitectura latinoamericana.

Los intentos más acabados de objetivos similares pueden observarse en los Seminarios de Arquitectura Latinoamericana y la gestación de sus «Mesas de Revistas» en cuyo camino se acreditan vínculos y esfuerzos comunes, valorizando la trascendencia editorial de esta temática. El establecimiento de una plataforma virtual en el espacio informático a partir de la reunión en 2009 en Panamá, bajo la denominación de «Asociación de Revistas Latinoamericanas de Arquitectura» ${ }^{[5]}$, dio comienzo a la cristalización del espíritu editorial de los SAL adelantado en 1985: «el reconocimiento de la arquitectura latinoamericana a través de sus propios medios de difusión». Así surgió ArLA. Asociación DE ReVISTAS LATINOAMERICANAS DE ARQUITECTURA, una propuesta que establece una sede virtual común en la web, desde la cual las revistas puedan presentarse como cuerpo colectivo que promueva el fácil acceso a la información, difunda sus contenidos, promueva la difusión de la arquitectura de nuestros países, entre varios otros objetivos. El objetivo de esta red permitirá establecer un conocimiento actualizado y un contacto permanente, compartir procesos editoriales que permitan estructurar un sistema y base de datos de contenidos, temáticas, proyectos, etc., que constituyan herramientas útiles a los asociados.

Con mayor razón, quienes en las páginas de las revistas de arquitectura asomamos a un mundo infinito de ideas, somos deudores de una actualización permanente y esta plataforma que nos congrega pareciera constituirse en el espacio de reunión adecuado para establecer una única voz que permita compartir problemáticas y facilitar soluciones.

El desafío de este siglo XXI resulta entonces intentar completar y profundizar la visión de la arquitectura continental a través de la revisión de esas páginas, un reto que sin dudas nos permitirá comprender la arquitectura del siglo xx a través de esa fuente inagotable e insustituible que fueron (y deben continuar siendo) las revistas de nuestra profesión.

\section{BIBLIOGRAFÍA}

Bouvier, Béatrice. «Répertoire des périodiques $d^{\prime}$ architecture en langue française, publiées entre 1800 et 1970, en France et dans ses anciennes colonies, en Suisse et en Belgique». En: publication papier dans la collection Etudes et rencontres de l'Ecole nationale des Chartres. Accompagné des actes de la journée d'étude du 2 juin 2000: «Presse architecturale (XVIIIle-XXe siècle) recherche d'une méthode $d^{\prime}$ analyse critique». Paris, École des Chartres, 2001.

Chartier, Roger. Las múltiples formas de leer y escribir. ADN Cultura, La Nación, 11 de octubre, 2008 [en: http://adncultura. lanacion.com.ar/nota.asp]

Gutiérrez, Ramón. «Architectural Journals and the Means for Discourse in Latin America». En: Design Book Review, 32-33 (printemps eté 1994), 1994, pp. 22-33.

Gutiérrez, Ramón. «Le reviste di architettura e I'architettura moderna in Iberoamerica». Casabella, 1995, 621, pp. 38-39.

Gutiérrez, Ramón. «Revista estadounidense para el consumo latinoamericano». Anales del Instituto de Investigaciones Estéticas. Universidad Nacional Autónoma de México, 2008, 92, pp. 245-250.

Gutiérrez, Ramón; Martín, Marcelo. Bibliografía iberoamericana de revistas de arquitectura y urbanismo. Madrid, Ediciones de las Universidades de Alcalá y Valladolid, Instituto Español de Arquitectura, Consejo Académico lberoamericano, 1993.

Gutiérrez, Ramón; Méndez, Patricia et al Revistas de Arquitectura de América Latina. 1900-2000. San Juan de Puerto Rico, Universidad Politécnica de Puerto Rico, 2001.

Leniaud, Jean Michel; Bouvier, Béatrice. Les périodiques d'architecture. XVIIle- XXIe siècle. Recherche d'une méthode critique d'analyse. Paris, Études et Rencontres de L'école des Chartres, 2001.

Serna, M.; David, E. «Sobre los textos en la Arquitectura Latinoamericana». En: Revista de la Universidad Católica Boliviana, Ciencia y Cultura, 2000, 6, pp. 19-37.

Sorli Rojo, Angela; Fernández Caballero, D.; Mochón Bezares, G.; Azorín López, V. «Las publicaciones periódicas de construcción en España y su importancia en la transferencia de conocimientos». En:

[5] Propuesta planteada por el arquitecto Hernán Ascui Fernández, de la revista chilena Arquitecturas del Sur de la Universidad del Bío-Bío. 
Actas de las II Jornadas de Investigación en Construcción, mayo de 2008 [en: http:// digital.csic.es/bitstream/10261/6236/1/ IIJIC Sorli.pdf].

Tarcus, Horacio. Las revistas culturales argentinas (1890-2006). Centro de Documentación e Investigación de la Cultura de Izquierda en la Argentina, 2007, 3.

Varela, Mirta. «Medios de comunicación e industrias culturales: historias nacionales y problemas globales». Documento presentado en el XXX Congresso Brasileiro de Ciencias da Comunicação. Intercom - Sociedad Brasileira de Estudios Interdisciplinares de Comunicação, São Paulo, 2007.
6. Ediciones digitales de Arquitectura México (1938-1978) y de TraZa (1983-1986) dentro del proyecto "Raíces DigitaL, Fuentes para la historia de la Arquitectura Moderna" de la UNAM.
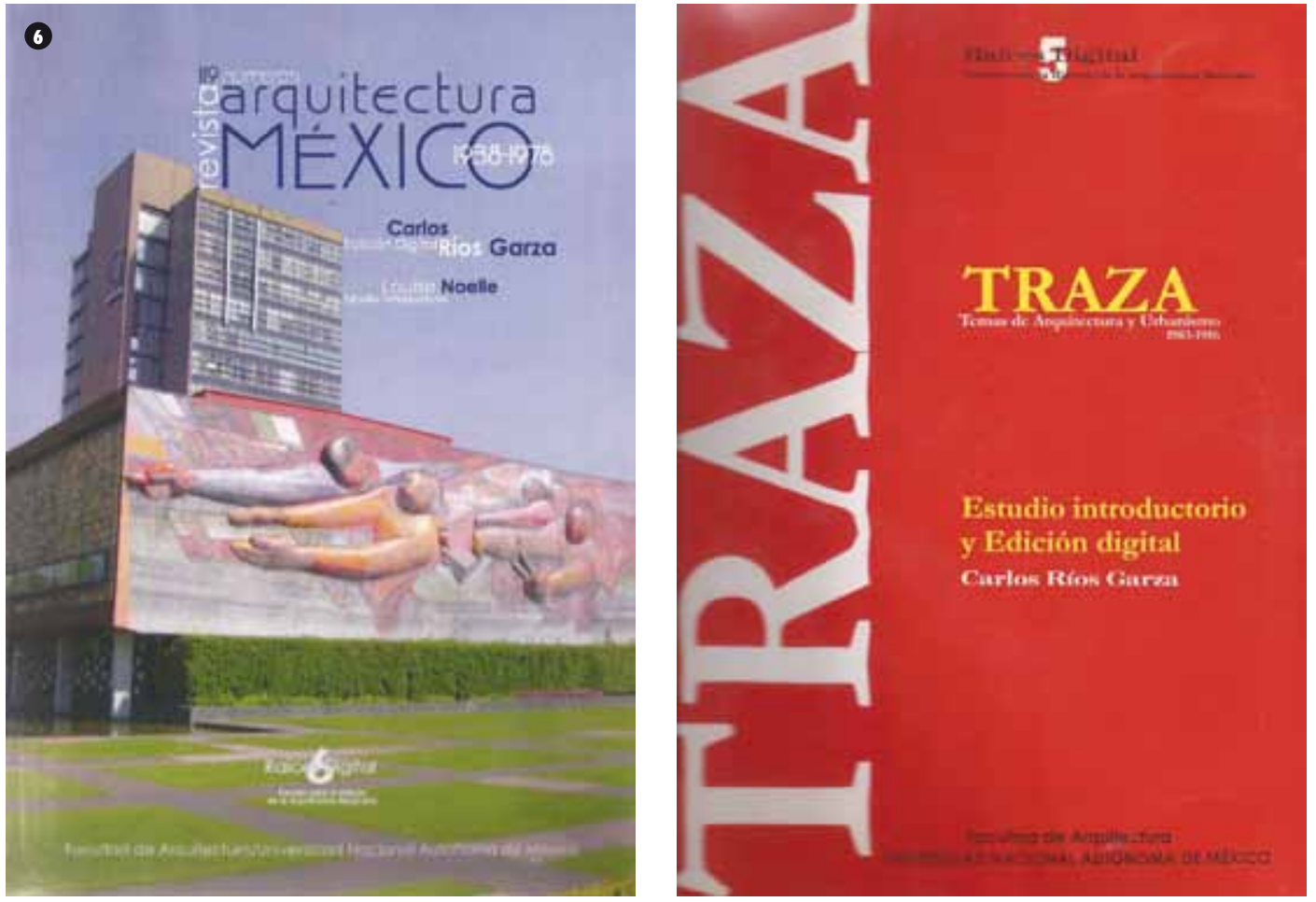